\title{
Corrigendum
}

\section{Corrigendum to "A Review of Collagen Cross-Linking in Cornea and Sclera"}

\author{
Xiao Zhang, ${ }^{1}$ Xiang-chen Tao, ${ }^{1}$ Jian Zhang, ${ }^{2}$ Zhi-wei Li, ${ }^{1}$ Yan-yun Xu, \\ Yu-meng Wang, ${ }^{1}$ Chun-xiao Zhang, ${ }^{1}$ and Guo-ying $\mathrm{Mu}^{1}$ \\ ${ }^{1}$ Department of Ophthalmology, Shandong Provincial Hospital Affiliated to Shandong University, Jinan, Shandong 250000, China \\ ${ }^{2}$ Zibo Eye Hospital, No. 33-6, Songlingxi Street, Zichuan, Zibo, Shandong 255100, China \\ Correspondence should be addressed to Guo-ying Mu; mgyeyes@163.com \\ Received 18 July 2016; Accepted 9 August 2016 \\ Copyright (C) 2016 Xiao Zhang et al. This is an open access article distributed under the Creative Commons Attribution License, \\ which permits unrestricted use, distribution, and reproduction in any medium, provided the original work is properly cited.
}

In the article titled "A Review of Collagen Cross-Linking in Cornea and Sclera" [1], the first affiliation was incorrectly written as "Department of Ophthalmology, Shandong Provincial Hospital, Shandong University, Jinan, Shandong 250000, China," while the correct address is "Department of Ophthalmology, Shandong Provincial Hospital Affiliated to Shandong University, Jinan, Shandong 250000, China." The corrected affiliations are shown above.

\section{References}

[1] X. Zhang, X.-c. Tao, J. Zhang et al., "A review of collagen crosslinking in cornea and sclera," Journal of Ophthalmology, vol. 2015, Article ID 289467, 12 pages, 2015. 


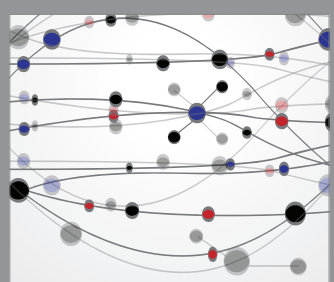

The Scientific World Journal
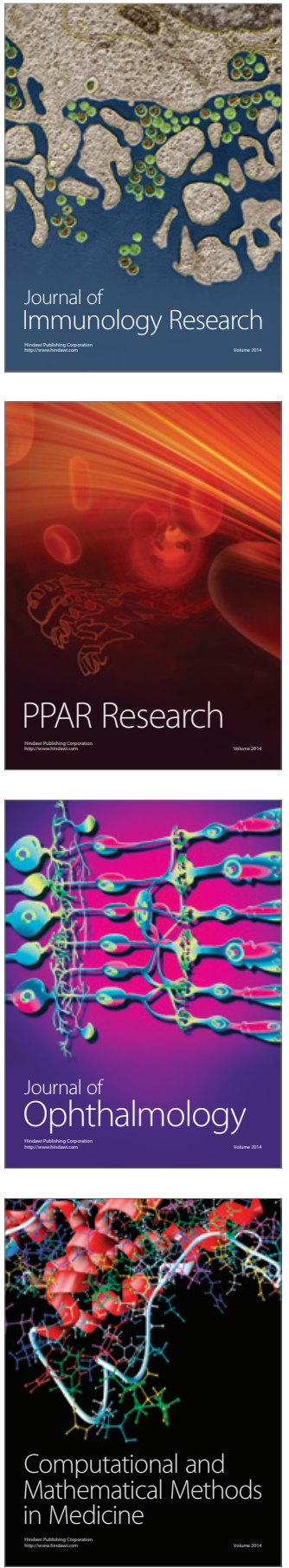

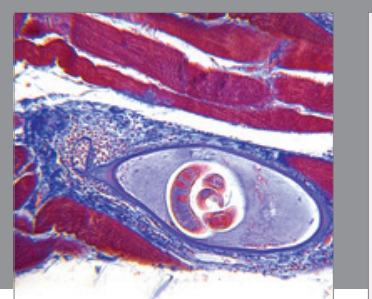

Gastroenterology Research and Practice

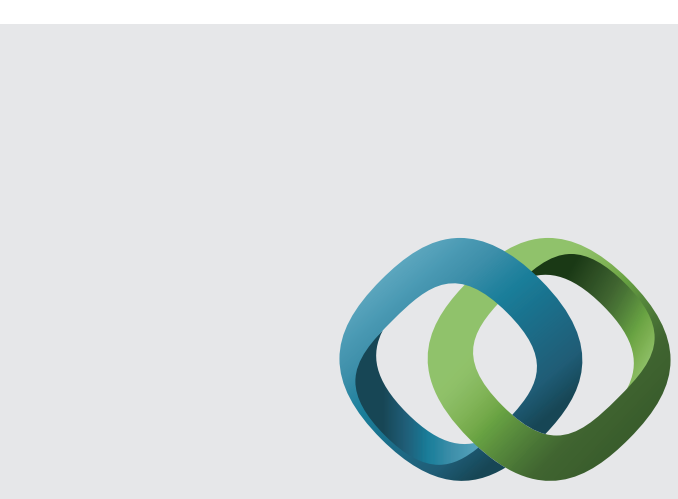

\section{Hindawi}

Submit your manuscripts at

http://www.hindawi.com
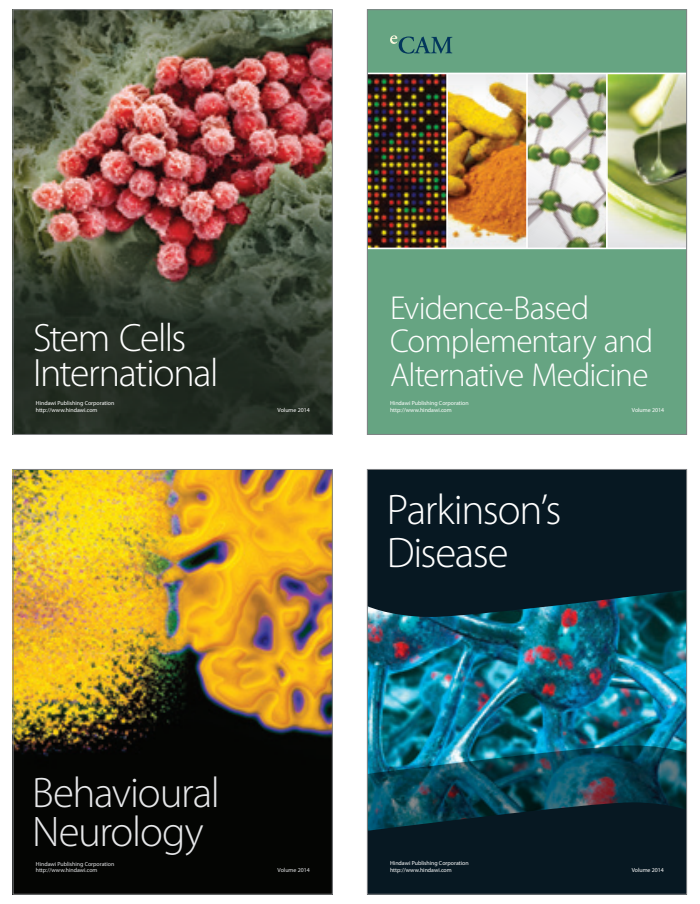
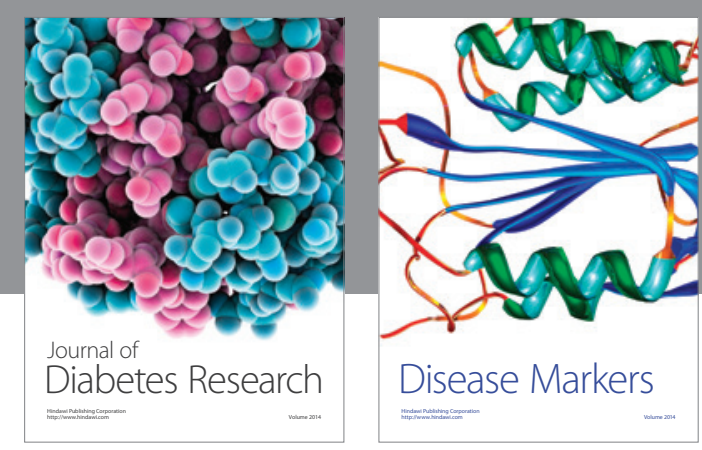

Disease Markers
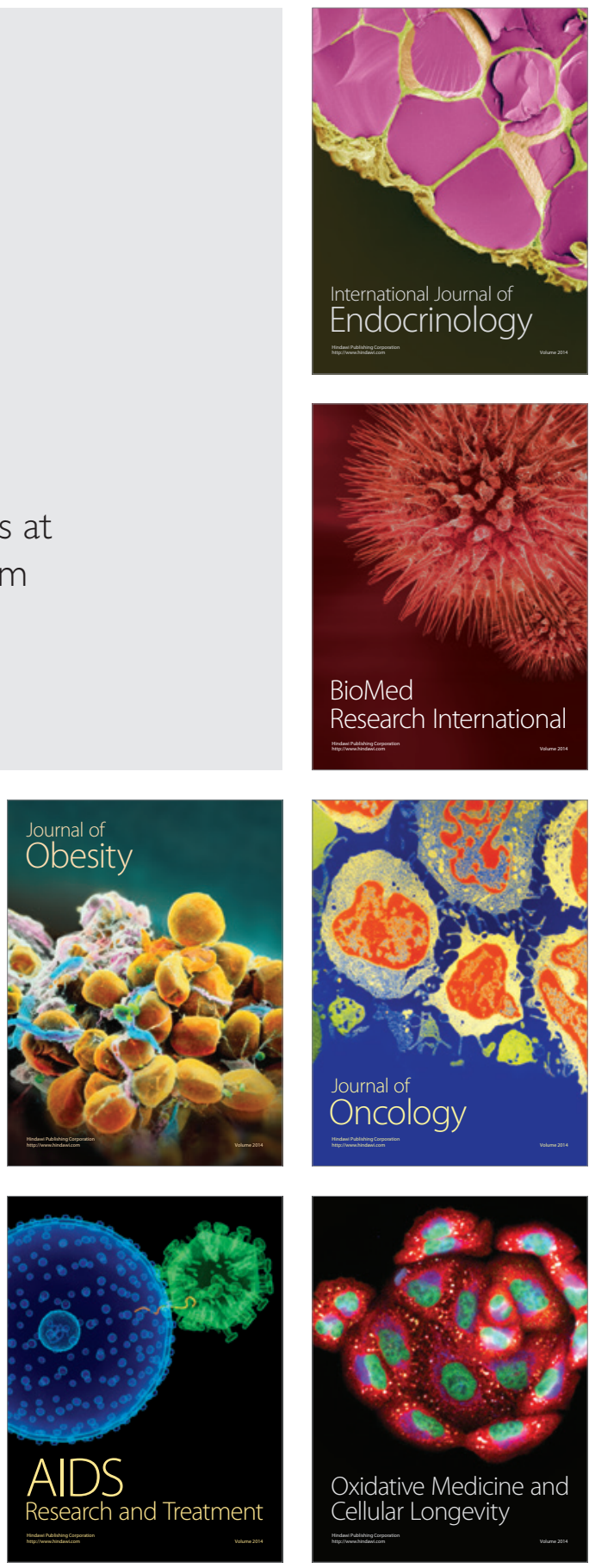